\section{Regulation of E-box DNA binding during in vivo and in vitro activation of rat and human hepatic stellate cells}

\author{
K J Vincent, E Jones, M J P Arthur, D E Smart, J Trim, M C Wright, D A Mann
}

under conditions of alcohol abuse and viral infection and leads to accumulation of excess extracellular matrix in the form of scar tissue eventually resulting in cirrhosis. ${ }^{1-5}$ It is therefore important to study the programme of molecular events that control HSC activation so as to understand how the processes of normal and pathological wound healing of the liver are controlled. We have previously used the cell culture model of HSC activation to describe the persistent induction of proteins belonging to the AP-1 and nuclear transcription factor $\kappa \mathrm{B}(\mathrm{NF} \kappa \mathrm{B})$ families which regulate transcription of important profibrogenic proteins such as tissue inhibitor of metalloproteinases 1 and intracellular adhesion molecule $1 .^{67}$ This type of analysis can be applied to other transcription factors including those belonging to the basic helix-loop-helix (bHLH) family of proteins which have been shown to act as master transcriptional regulators of the growth and differentiation of mammalian cells. ${ }^{8-10}$

The DNA binding recognition sequence of bHLH proteins is the E-box (CANNTG) which is present in the promoter and enhancer regions of numerous developmentally regulated genes. ${ }^{11}{ }^{12}$ bHLH proteins exist as two distinct families, class $\mathrm{A}$ and $\mathrm{B}$, that bind to the E-box in the form of homo- and heterodimers. ${ }^{11-14}$ Class A bHLH proteins are often referred to as "E" proteins and are ubiquitously expressed. By contrast, class B proteins tend to be tissue or cell specific and include the myogenic transcription factors exemplified by the prototype factor MyoD which controls skeletal muscle differentiation. ${ }^{9}$ A third set of related bHLH proteins known as inhibitors of differentiation (Id) lack a DNA binding domain and can act as inhibitors of the class A and class B proteins. ${ }^{15}$ As it is conceivable that the dramatic phenotypic change associated with activation of HSC may be in part regulated by bHLH transcription factors, we have investigated changes in E-box DNA binding activity during in vitro and in vivo activation of rat and human HSCs.

Abbreviations used in this paper: HSC, hepatic stellate cell; $\alpha$-SMA, $\alpha$-smooth muscle actin; NFкB, nuclear transcription factor $\mathrm{\kappa B}$; bHLH, basic helix-loop-helix; Id, inhibitor of differentiation; EMSA, electrophoretic mobility shift assay; SDS-PAGE, sodium dodecyl sulphate-polyacrylamide gel electrophoresis; RT-PCR, reverse transcription-polymerase chain reaction; PDGF, platelet derived growth factor. 


\section{Methods}

ISOLATION AND CULTURE OF RAT AND HUMAN HSCs

Primary rat HSCs were isolated from the livers of normal or carbon tetrachloride treated six month old $500 \mathrm{~g}$ male Sprague-Dawley rats, as previously described. ${ }^{16}$ Induction of acute liver damage in rats was achieved by administration of carbon tetrachloride by intraperitoneal injection with $0.2 \mathrm{ml} / 100 \mathrm{~g}$ sterile carbon tetrachloride in a 1:1 ratio with olive oil, as described previously. ${ }^{17}$ Human HSCs were isolated from adult male human liver following partial hepatectomy performed on consenting patients undergoing resection for secondary cancer originating in the small intestine. Only those liver tissues judged as non-cancerous by local pathologists were used for cell preparations. Sequential perfusion with pronase and collagenase was carried out as described for isolation of rat HSC prior to separation of HSC over an $11.5 \%$ Optiprep gradient. Isolated rat and human HSCs were cultured onto plastic in Dulbecco's modified Eagle's medium supplemented with $16 \%$ fetal calf serum and maintained at $37^{\circ} \mathrm{C}$ in an atmosphere of $5 \%$ $\mathrm{CO}_{2}$.

ELECTROPHORETIC MOBILITY SHIFT ASSAY (EMSA) Preparation of nuclear extracts and conditions for electrophoretic mobility shift assay (EMSA) reactions were as previously described. ${ }^{78}$ Oligonucleotides used in the EMSA reactions were as follows: "mE-box", sense strand (S) 5'CGCCGACCACGTGGTCCCTC-3' and anti-sense strand (A) 5'-GAGGGACCACGTG GTCGGCG-3'; “ “ $\Delta$ mE-box” (mutant mE-box), (S) 5'-CGCCGACATCGCTGTCCCTC-3' and (A) 5'-GAGGGACAGCGATGTCG GCG-3'; “ $\alpha$-SMA E-box”, (S) 5'-GATCATAA GCAGCTGAACTGCC-3' and (A) 5'-GGC AGTTCAGCTGCTTATGATC-3'; “ $\Delta \alpha$-SMA E-box", (S) 5'-GATCATAAGTAGGCGAACT GCC-3' and (A) 5'-GGCAGTTCGCCTA CTTATGATC-3'; "m $\alpha$-SMA E-box" ( $\alpha$-SMA E-box core with mE-box flanking sequences), (S) 5'-CGCCGACCAGCTGGTCCCTC-3' and (A) 5'-GAGGGACCAGCTGGTCGG CG-3'. Supershift/antibody interference EMSA reactions were performed by incubation of EMSA reaction mixtures for 16 hours at $4^{\circ} \mathrm{C}$ with $4 \mu \mathrm{g}$ of polyclonal antibody prior to electrophoresis. All polyclonal antibodies were purchased from Santa Cruz (Autogen Bioclear, UK) and included anti-MyoD (M-318), antiMyoD (C-20), anti-myogenin (M-225), anti-cMyc (N-262), anti-Max (C-124), and antiMad-1 (FL-221).

RNA EXTRACTION AND NORTHERN BLOTTING RNA was prepared from rat HSC as previously described. ${ }^{16}$ Aliquots of $20 \mu \mathrm{g}$ total RNA were separated on denaturing $1 \%$ agarose gels, transferred to nylon filters (Hybond N, Amersham) by capillary elution, and fixed by exposure to UV light $(254 \mathrm{~nm})$ for five minutes. Filters were probed with an oligonucleotide (5'-CGGGGCCTATCAAGTCTGT GTCCCGGAGTGGCGGCGATAGTAGCT CCAT-3') designed to hybridise to the first 49 coding nucleotides of the MyoD cDNA sequence; for Id 1 with a cDNA for mouse Id 1 (a kind gift of Dr John Norton, Manchester, UK); and for $\beta$-actin with a cDNA probe for rat $\beta$-actin. All DNA probes used for northern blotting were labelled with $\left[\alpha-{ }^{32} \mathrm{P}\right]$ ATP by random priming. Following hybridisation for 16 hours at $42^{\circ} \mathrm{C}$, filters were washed three times for 15 minutes at $50^{\circ} \mathrm{C}$ in $0.2 \times \mathrm{SSC}+0.2 \%$ sodium dodecyl sulphate (SDS). Filters were subjected to autoradiography using preflashed Fuji AR film for $24-72$ hours at $-70^{\circ} \mathrm{C}$.

SODIUM DODECYL SULPHATE

(SDS)-POLYACRYLAMIDE GEL ELECTROPHORESIS (PAGE) AND IMMUNOBLOTTING

Equal quantities $(20 \mu \mathrm{g})$ of nuclear protein extract were subjected to electrophoresis on $4 \%$ stacking $12.5 \%$ SDS-PAGE gels which were processed for immunodetection analysis as previously described. ${ }^{6} 7$

REVERSE TRANSCRIPTION-POLYMERASE CHAIN REACTION (RT-PCR) DETECTION OF MyoD mRNA. Total cellular RNA $(1 \mu \mathrm{g})$ extracted from seven day activated rat HSCs was reverse transcribed using an antisense MyoD primer 5'CTGCAGACCTTCAATGTAGC-3'. RT reaction mixtures were then directly used for PCR using sense MyoD primer (5'AAGACCACTAACGCTGATCG-3'). A PCR product obtained at $1 \mathrm{mM} \mathrm{Mg}^{2+}$ was blunt ended by incubation with Vent polymerase prior to agarose gel electrophoresis and extraction of the excised product using the QIAquick gel extraction kit (Qiagen, UK). The purified blunt end PCR product was then ligated into the $E c o R V$ site pcDNA3 (Invitrogen, UK) and transformed into Escherichia coli. Six independent clones containing the PCR product were then sequenced.

\section{Results}

ACTIVATION INDUCED MODULATION OF E-box DNA BINDING ACTIVITIES IN RAT AND HUMAN HSCs

Nuclear extracts from freshly isolated and cultured rat HSCs were probed for E-box DNA binding activities by EMSA using a palindromic E-box sequence (mE-box) previously used to detect c-Myc DNA binding events. ${ }^{18} \mathrm{Nu}-$ clear extract from freshly isolated rat HSCs formed a single mE-box binding complex (C) of intermediate mobility on the gel (fig 1A, 1B). Nuclear extracts from HSCs cultured across time points ranging from three hours to 14 days lacked the ability to form complex C (fig 1A) but instead formed a pair of low mobility complexes (A and B). Complex A was routinely more abundant than complex B with the latter complex being barely visible in some experiments. Figure 1A also shows the result of combining equal quantities of nuclear extract from freshly isolated and 14 day activated HSCs in the same EMSA reaction mix $(\mathrm{FI}+14 \mathrm{~d})$. In this latter reaction, complex C was assembled at a level similar to that observed in reactions containing nuclear protein from freshly isolated HSCs alone; in 

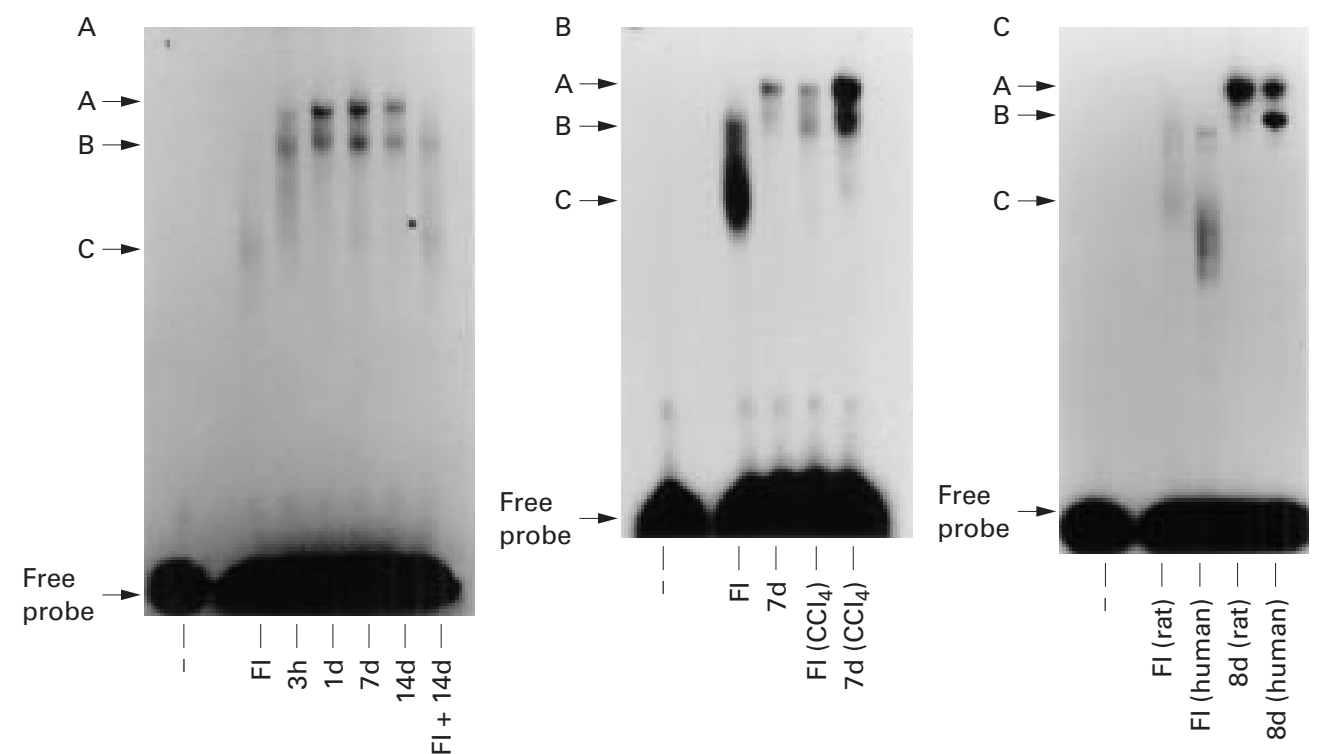

Figure 1 Analysis of E-box DNA binding activities in primary rat and human hepatic stellate cells (HSCs). (A) Modulation of E-box DNA binding in cultured rat HSCs. Nuclear extracts (10 $\mu \mathrm{g})$ prepared from freshly isolated (FI) and cultured (three hours to 14 days) rat HSCs were used to detect E-box DNA binding activities by EMSA. A control track (-) lacking nuclear extract was included on the far left hand side of the gel. FI+14 days shows the effects of adding equal quantities (10 $\mu \mathrm{g}$ of each) of nuclear extract into the same EMSA reaction mixture. $A, B$, and $C$ denote retarded E-box DNA:protein binding complexes. (B) Modulation of E-box DNA binding during in vivo activation of rat HSCs. Control and carbon tetrachloride (CCl) treated rats (48 hours) were used to prepare HSCs which were either harvested (FI and FI $(C C l))$ or cultured for seven days $(7 d$ and $7 d(C C l))$ prior to preparation of nuclear extracts; $10 \mu g$ of each extract were used for electrophoretic mobility shift assay (EMSA). (C) Comparison of rat and human HSC E-box DNA binding activities: $10 \mu \mathrm{g}$ of nuclear extract prepared from freshly isolated (FI) or eight day cultured (8d) rat and human HSCs were used in EMSA assays. All gels are representative of at least three independent experiments.

contrast, levels of complexes $\mathrm{A}$ and $\mathrm{B}$ were substantially diminished relative to reactions containing nuclear protein exclusively from activated HSCs.

Many of the biochemical changes associated with in vitro activation of HSCs are faithful to changes observed in animal models of HSC activation and hepatic fibrosis. ${ }^{2319}$ To determine if this was also the case for E-box DNA binding activities, nuclear extracts were prepared from freshly isolated HSCs purified from the livers of rats 48 hours after intraperitoneal injection of carbon tetrachloride, an inducer of HSC activation. ${ }^{17}$ Figure $1 \mathrm{~B}$ shows that nuclear extracts prepared from these cells did not assemble complex $\mathrm{C}$ but instead formed two complexes with identical mobilities to complexes A and B observed in cultured HSCs isolated from control rats. This result was reproducible using nuclear extracts purified from several carbon tetrachloride treated rats including animals exposed to the chemical for three weeks (data not shown). Culturing of HSCs derived from carbon tetrachloride treated rats led to an increase in the abundance of complexes A and B to levels exceeding those observed in control HSCs cultured for a similar period of time. We conclude that in vivo activation of HSCs results in similar modulation of E-box DNA binding activities to those observed in vitro. E-box DNA binding activities were also comparable in human and rat HSCs (fig 1C), indicating the generality of the activation induced shift in mobility of protein:DNA interactions on the E-box sequence.
HSC mE-box DNA BINDING ACTIVITIES ARE SEQUENCE SPECIFIC

To test specificity of HSC E-box protein:DNA interactions, competition EMSA reactions were performed using an excess of unlabelled specific (E-box) or non-specific (SP1) double stranded oligonucleotides. Assembly of complexes A, B (fig 2A), and C (fig $2 \mathrm{~B}$ ) was effectively competed out using a 20 -fold or 80 -fold excess of a specific competitor; in contrast, no competition was observed using up to an 80-fold excess of non-specific competitor. Requirement for an intact E-box to form protein:DNA complexes on the mE-box was demonstrated by lack of competition by a mutant mE-box oligonucleotide $(\Delta \mathrm{mE}$-box) in which the core E-box sequence 5'CACGTG-3' was changed to 5'-ATCGCT-3' (fig 3A). Direct binding studies showed that an E-box derived from the $\alpha$-SMA gene promoter (" $\alpha$-SMA E-box") was only able to assemble very weak protein:DNA complexes with nuclear extracts from activated HSCs (data not shown). Since these complexes appeared to be of distinct mobilities to those obtained with the mE-box, competition EMSA reactions were employed to establish if proteins binding to the mE-box could also bind to the $\alpha$-SMA E-box. As shown in fig 3A, neither a wild type nor a mutant $\alpha$-SMA E-box could compete for binding with $\mathrm{mE}$-box. The sequence difference between the core E-boxes of the mE-box (5'-CACGTG-3') and $\alpha$-SMA E-box (5'CAGCTG-3') probes was the central dinucleotide pair. When the central CG pair of the mE-box was mutated to a GC pair the resultant 


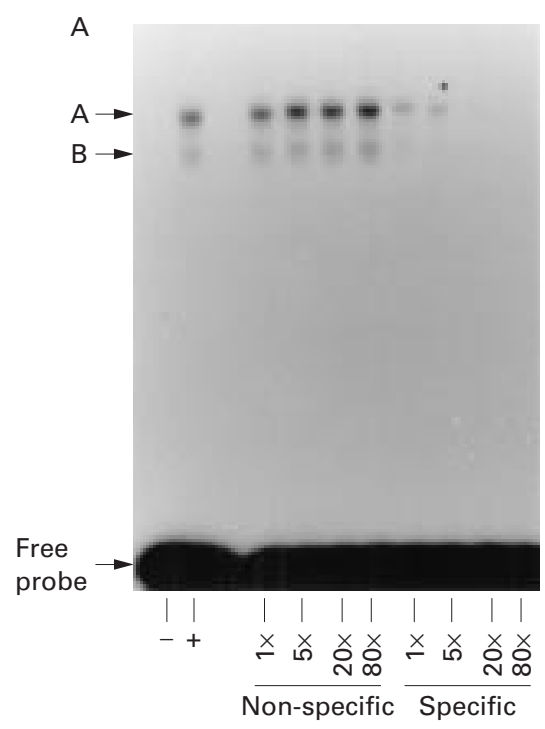

B

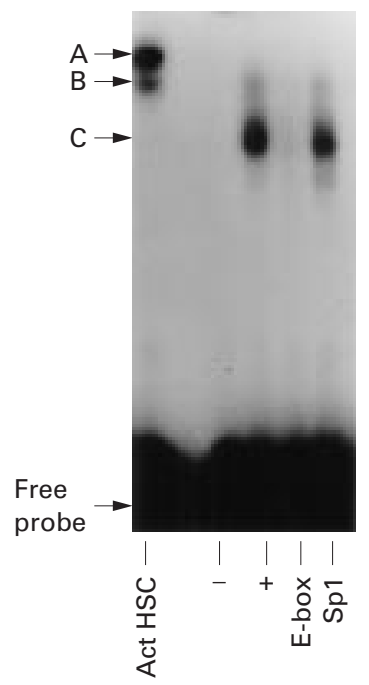

Figure 2 Determination of the specificity of hepatic stellate cell (HSC) E-box DNA:protein complexes. (A) Competition electrophoretic mobility shift assay (EMSA) was performed using nuclear extracts from activated rat HSCs and up to an 80-fold excess of specific (E-box) or non-specific (Sp1) unlabelled double stranded oligonucleotides as competitors. (B) Competition EMSA performed with nuclear extracts from freshly isolated rat HSCs using an 80-fold excess of specific (E-box) or non-specific (Sp1) oligonucleotides. Both gels are representative of at least three independent experiments.

E-box (ma-SMA E-box) was able to compete for mE-box binding (fig 3B). Direct binding studies also demonstrated that the chimeric ma-SMA E-box was able to assemble complexes $\mathrm{A}$ and $\mathrm{B}$ from activated HSC nuclear extracts (data not shown). These data indicate that flanking sequences of the E-box motif have a strong influence on its ability to specifically interact with HSC derived bHLH proteins.
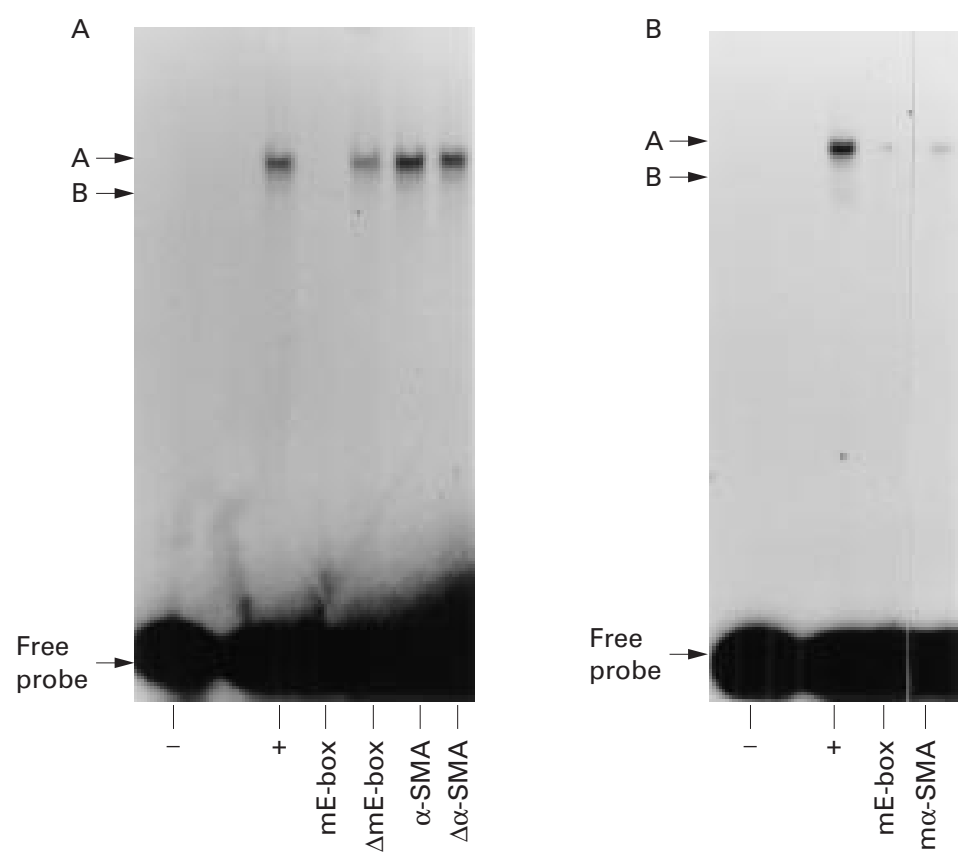

Figure 3 Sequence requirements for $m E$-box DNA binding. (A) Competition assay between $m E$-box and $a$-smooth muscle actin ( $a-S M A)$ E-box. Nuclear extract from activated rat hepatic stellate cells (HSCs) was initially incubated in the presence of an 80-fold excess of unlabelled wild type $m E$-box, mutant (4) $m E-b o x, a-S M A E-b o x$, or mutant (4) a-SMA E-box prior to incubation with radiolabelled wild type $m E$-box probe. (B) Competition assay between $m E-$ box and ma-SMA E-box. Nuclear extract from activated rat HSCs was initially incubated with an 80-fold excess of unlabelled wild type $m E$-box or chimeric ma-SMA E-box oligonucleotides prior to incubation with radiolabelled $m E-b o x$ probe. Gels are representative of two independent experiments.

IDENTIFICATION OF bHLH PROTEINS BINDING TO THE mE-box IN ACTIVATED HSC

In order to identify HSC expressed bHLH proteins that interact with the mE-box, a panel of antibodies recognising a variety of bHLH proteins was used in supershift/binding interference experiments. Polyclonal antibodies recognising Max, Mad-1, or the myogenic transcription factor myogenin failed to generate supershift complexes and did not interfere with complex formation (fig 4). In contrast, a polyclonal antibody (M-318) recognising full length MyoD protein caused a loss of complexes A and B. A second polyclonal antibody (C-20) recognising only the terminal 20 amino acids at the carboxy end of $\mathrm{MyoD}$ failed to block complex A and B assembly. A polyclonal antibody recognising $\mathrm{c}-\mathrm{Myc}$ occasionally gave rise to a weak interference of complex A and B formation. Similar supershift studies were also performed on complex $\mathrm{C}$ but these experiments failed to generate significant reactivity with either the M-318 or C-20 anti-MyoD antibodies or any of the other anti-bHLH reagents used against complexes $\mathrm{A}$ and $\mathrm{B}$ (Vincent, data not shown). The identity of the protein components of complex $\mathrm{C}$ therefore remain unknown.

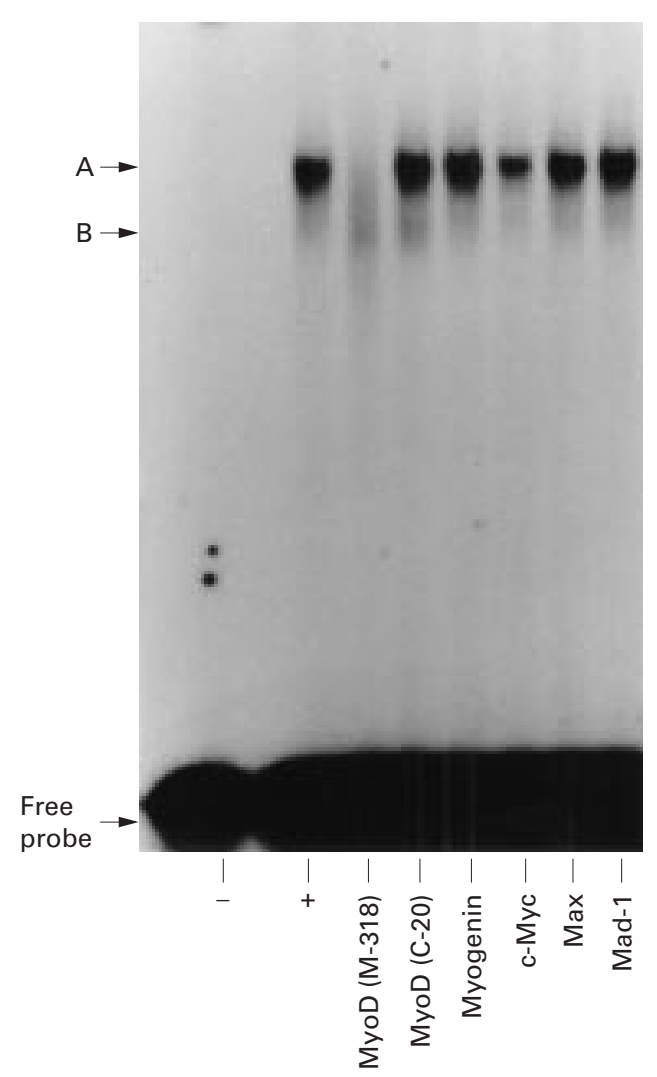

Figure 4 Reactivity of hepatic stellate cell (HSC) $m E$-box complexes with an anti-MyoD antibody. Electrophoretic mobility shift assay (EMSA) reactions consisting of nuclear extract from activated rat HSCs and wild type $m E$-box probe were incubated for 16 hours at $4^{\circ} \mathrm{C}$ either unsupplemented (+) or supplemented with polyclonal antibodies recognising $M y o D$ (M318 or $C-20)$, myogenin, $c-M y c, M a x$, and Mad-1. The gel is representative of two independent experiments. 
A

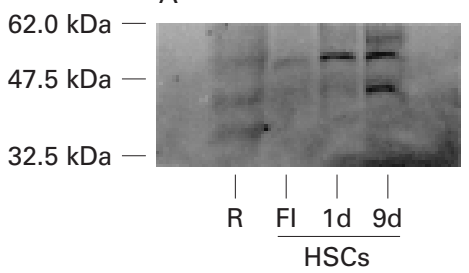

C

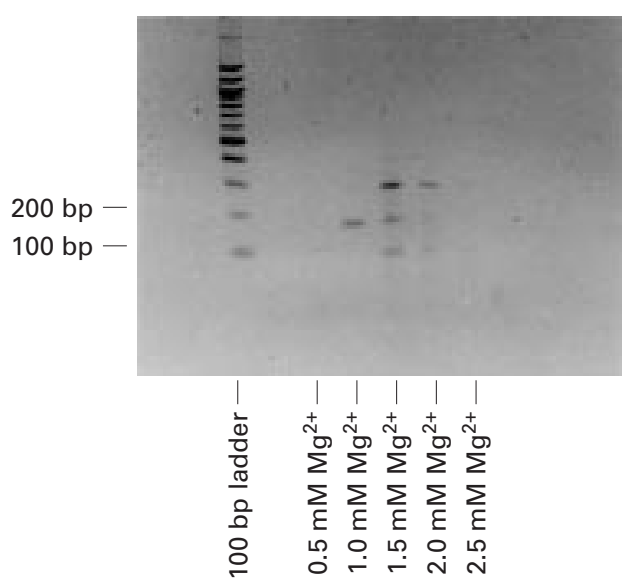

B
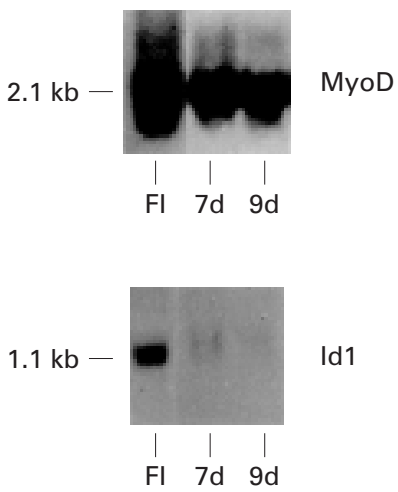

Id1

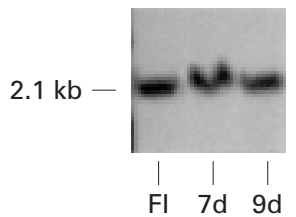

$\beta$-actin

Figure 5 Regulation of MyoD expression in rat hepatic stellate cells (HSCs). (A) Immunoblot detection of MyoD protein expression in rat HSCs. Sodium dodecyl sulphate-polyacrylamide gel electrophoresis and immunoblotting were performed as described in methods using equal quantities of nuclear protein extracts from RAW264.7 macrophages (R), freshly isolated rat HSCs (FI), and rat HSC cultured for one (1d) or nine (9d) days on plastic. (B) Northern blot detection of MyoD $m R N A$ in rat HSCs. Northern blotting was performed as described in methods using equal quantities of whole cell RNA extracted from freshly isolated (FI) rat HSCs and rat HSCs cultured on plastic for either seven (7d) or nine (9d) days. Filters were probed with radiolabelled cDNA probes for MyoD, Id1, and $\beta$-actin. (C) Reverse transcription-polymerase chain reaction (RT-PCR) detection of MyoD mRNA in activated rat HSCs. Following reverse transcription, equal quantities of cDNA were amplified using primers spanning nucleotides 468 to 642 of the MyoD cDNA sequence and in the presence of 0.5 to $2.5 \mathrm{mM} \mathrm{MgCl}$. The single $174 \mathrm{bp}$ PCR product obtained using $1.0 \mathrm{mM} \mathrm{MgCl}$, was subcloned into pcDNA3 and confirmed as exon 1 derived MyoD sequence by DNA sequence analysis.

EXPRESSION OF MyoD AND ITS INHIBITOR Id1 IN PRIMARY RAT HSCs

Ability of the anti-MyoD antibody M-318 to interfere with mE-box interactions suggested that primary HSCs might express the myogenic transcription factor. This was initially confirmed by immunoblotting using the M-318 antibody. Figure 5A shows that rat HSCs express a major immunoreactive species with an apparent molecular weight of approximately $50 \mathrm{kDa}$ in agreement with a previous report. ${ }^{20}$ Whereas expression was only weak in freshly isolated HSCs, culture activation for one or nine days resulted in a significant increase in the $50 \mathrm{kDa}$ species. A macrophage cell line RAW264. $7^{21}$ lacked detectable immunoreactivity with M-318 demonstrating cell type specific expression of MyoD. Similar results were obtained using the anti-MyoD antibody C20 (Vincent and Mann, unpublished data). Northern blot of total RNA with an oligonucleotide that hybridises to MyoD mRNA demonstrated equal levels of expression of a $2.1 \mathrm{~kb}$ mRNA in freshly isolated and culture activated rat HSC (fig 5B). Expression of MyoD RNA in activated rat HSCs was confirmed by RT-PCR analysis using primers that specifically amplify nucleotides 468 to 642 of the MyoD cDNA sequence inclusive of the highly conserved bHLH motif. ${ }^{22}$ A single 174 bp RT-PCR product obtained at a $\mathrm{MgCl}_{2}$ concentration of $1 \mathrm{mM}$ was confirmed by DNA sequence analysis to have $100 \%$ sequence identity with rat MyoD (fig 5C). Northern blots were also probed with cDNA for the prototypic inhibitory bHLH protein Id $1 .{ }^{15}$ In contrast with MyoD, levels of Id $1 \mathrm{mRNA}$ were significantly attenuated during HSC activation with high levels in freshly isolated cells being downregulated to lower but still detectable levels in culture activated cells (fig 5B). Reprobing of northern blot filters with a $\beta$-actin cDNA confirmed a roughly equal loading of RNA between samples. These latter results raise the possibility that Id 1 may be the factor in freshly isolated HSCs that is able to inhibit assembly of complex A and B by freshly isolated HSCs (fig 1A).

\section{Discussion}

In this study we have demonstrated that primary HSCs express the myogenic transcription factor MyoD and have shown that the E-box DNA binding of this class B bHLH protein is induced during in vitro and in vivo activation of rat and human HSCs. MyoD is the prototypic member of a family of transcription factors that regulate expression of muscle specific genes during development. ${ }^{9}$ Expression of MyoD DNA binding activity in activated HSCs implicates the transcription factor in the regulation of genes controlling myogenic properties of these cells. ${ }^{192-25}$ In an earlier report, MyoD expression was demonstrated in a liver stellate cell line by histochemical staining. ${ }^{23}$ However, it is still to be demonstrated that the 
cell line, 2G, used in this study provides an accurate cellular representation of primary activated HSCs. In addition, it has been reported that caution should be exercised when interpreting immunostaining results with antiMyoD antibodies as they can cross react with currently unknown cellular proteins to generate artefactual data. ${ }^{26}$ Our study therefore substantially advances this earlier report by demonstrating expression of MyoD mRNA, protein, and DNA binding activity in primary HSCs.

As we have previously shown with $\mathrm{AP}-1$ and $\mathrm{NF \kappa B},{ }^{67}$ activation of HSCs on tissue culture plastic is accompanied by changes in the DNA binding activity of proteins that recognise the E-box regulatory motif. Two studies have previously documented changes in E-box DNA binding during HSC activation, however neither study indicated a role for MyoD. ${ }^{27}{ }^{28}$ Lee et al demonstrated induction of c-Myb:E-box complexes in activated rat HSCs using the $\alpha$-SMA E-box sequence described in our study ${ }^{27}$ More recently, Weiner and colleagues ${ }^{28}$ described the requirement for E-box sequences in the activation of the promoter of the mannose 6-phosphate receptor gene in response to platelet derived growth factor (PDGF) treatment of activated rat HSCs. In this latter study the E-box element conferring PDGF responsiveness formed a single complex with HSC nuclear extracts that was induced by HSC activation and PDGF treatment of serum deprived HSCs. The bHLH proteins responsible for this E-box binding activity were not described. In the current study, we have demonstrated that in vitro and in vivo activation of rat HSCs and in vitro activation of human HSCs is accompanied by induction of low mobility E-box binding complexes (A and B) that are at least in part composed of MyoD containing bHLH dimers. Assembly of the MyoD:E-box complexes is therefore a general feature of HSC activation and as such is likely to be of functional significance in the regulation of the activated HSC phenotype.

The basis for our conclusion that the mE-box DNA:HSC protein complexes A and $B$ contain MyoD is the strict sequence specificity required for assembly of these complexes coupled with their reactivity with the antiMyoD polyclonal antibody M-318 that recognises full length MyoD. The MyoD containing complexes were assembled with the mE-box sequence but were not formed with the related $\alpha$-SMA E-box sequence. MyoD dimers have been reported to prefer E-box motifs that are palindromic and contain purines on the $5^{\prime}$ flanking side and pyrimidines on the 3' flanking side. ${ }^{11}$ The mE-box (5'-GACCACGTGGTC$3^{\prime}$ ) is palindromic and has purine rich 5 , flanking DNA and pyrimidine rich 3' flanking DNA; in contrast, the $\alpha$-SMA E-box (5'AAGCAGCTGAAC-3') is not palindromic and contains purine rich flanking sequences on both sides of the core E-box motif. The importance of the flanking sequences of the mE-box sequence was demonstrated by showing that a chimeric E-box (known as m $\alpha$-SMA E-box) consisting of the core sequence of
$\alpha$-SMA E-box and the flanking sequences of the mE-box were able to compete for protein binding with the original mE-box probe. This strict sequence specificity therefore supports our argument that MyoD is a constituent of the mE-box protein:DNA interactions induced during HSC activation. Further support was provided by the ability of the anti-Myo D antibody M-318 to inhibit assembly of complexes $\mathrm{A}$ and $\mathrm{B}$ while in contrast antibodies recognising a variety of bHLH proteins including the related myogenic transcription factor myogenin were without effect. However, a second MyoD antibody (C-20) raised against the carboxy terminal end of the protein also failed to recognise complexes $\mathrm{A}$ and $\mathrm{B}$. It is possible that conformational constraints in the MyoD:mEbox interaction disallowed recognition of the $\mathrm{C}$ terminal region of MyoD by C-20. The mE-box sequence resembles an extended palindromic E-box (CCACGTGG) present in the mouse and hamster genes encoding the pyrimidine biosynthetic enzyme carbomyl phosphate synthase which is required for the $S$ phase of the cell cycle. ${ }^{29}{ }^{30}$ Insertion of three tandem mE-box sites upstream of the herpes simplex virus thymidine kinase promoter resulted in a reduction of promoter activity in activated rat HSCs (Mann and Vincent, unpublished observation). It is therefore possible that one function of MyoD is to exert a negative influence on HSC proliferation. Other genes carrying extended palindromic mE-box like motifs include the rat CTL-1 gene (ACCACGTGGT) which encodes a choline transporter, ${ }^{31}$ the human I $\mathrm{I} B-\varepsilon$ gene (CCCACGTGGG), ${ }^{32}$ and the human voltage dependent calcium channel alpha IG subunit gene (GACCACGTGGTC). ${ }^{33}$

Induction of complex $\mathrm{A}$ and $\mathrm{B}$ formation during HSC activation was accompanied by loss of complex $\mathrm{C}$ and elevation of MyoD protein expression. As levels of MyoD mRNA were unchanged with HSC activation, we suggest that MyoD expression during HSC activation is likely to be controlled by a posttranscriptional mechanism. Increased rate of synthesis and/or a decreased rate of proteolytic degradation could result in elevation of steady state levels of MyoD. Alternatively, enhanced MyoD expression may be a consequence of induction of MyoD DNA binding activity as it has recently been demonstrated that MyoD bound to E-box containing DNA may be less susceptible to ubiquitin mediated degradation. ${ }^{34}$ This stabilising effect of DNA on MyoD can be abrogated by the inhibitory bHLH protein Id1 which blocks MyoD:DNA interactions. ${ }^{34}$ We have shown in the present study that culture activation of rat HSC is accompanied by a rapid and long term diminution of $\mathrm{Id} 1$ mRNA suggesting that levels of the inhibitor may be reduced in activated HSCs. As Id family proteins are relatively short lived factors, ${ }^{35}$ the observed diminution of Id 1 mRNA would result in a similar loss of Id 1 protein expression in activated HSCs. Id 1 is one of four bHLH proteins that lack the amino terminal basic region necessary for DNA binding yet retain the ability to dimerise with class $\mathrm{A}$ and class $\mathrm{B}$ 
bHLH factors and in doing so prevent their ability to form DNA binding homo- or heterodimers. ${ }^{15}$ Although Id 1 has highest affinity for class A proteins it can also bind avidly to a subset of class B proteins including MyoD. ${ }^{36}$ Hence in quiescent HSCs, high levels of Id1 would prevent formation of MyoD homo- and heterodimers and their association with DNA and favour ubiquitin mediated degradation. On activation, reduced levels of Id 1 would promote formation of MyoD dimers which would then be stabilised from proteolysis leading to raised steady state levels of MyoD protein in activated HSCs. We suggest that loss of Id1 expression will allow formation of complexes A and $\mathrm{B}$ by MyoD. It is also possible that changes in the DNA binding and/or expression levels of the protein components of complex $\mathrm{C}$ may also be required if formation of complexes $\mathrm{A} / \mathrm{B}$ and $\mathrm{C}$ are mutually exclusive. Downregulation of Id 1 has been linked with differentiation of skeletal muscle cells while forced expression of Id 1 has been shown to perturb the differentiation of a wide variety of cell types implicating Id 1 as a general inhibitor of cell differentiation. ${ }^{36}$ Our demonstration of a diminution of Id 1 mRNA expression with HSC activation suggests that Id1 may also function as a negative regulator of the transdifferentiation of HSCs. Future studies should now be directed towards determining a functional role for MyoD and Id1 in the regulation of HSC activation and the molecular pathology of liver fibrosis. These studies should include identification of HSC expressed genes that are targets for MyoD DNA binding.

This work was funded by grants provided by the Wellcome Trust, Hope (Wessex Medical Trust), and the UK Medical Research Council. KV was an MRC research student and DES is a recipient of a Wessex Medical Trust Jubilee PhD studentship. The authors thank Dr John Norton for his kind gift of Id 1 cDNA.

1 Friedman SL. The cellular basis of hepatic fibrosis. $N$ Engl $\mathcal{F}$ Med 1993;328:1828-35.

2 Friedman SL. Molecular regulation of hepatic fibrosis, an integrated cellular response to tissue injury. $f \mathrm{Biol}$ Chem 2000;275:2247-50.

3 Pinzani M. Hepatic stellate (ITO) cells: expanding roles for a liver-specific pericyte. $\mathcal{F}$ Hepatol 1995;22:700-6.

4 Minato Y, Hasumura Y, Takeuchi J. The role of fat-storing cells in Disse space fibrogenesis in alcoholic liver disease. cells in Disse space fibrogen
Hepatology 1983;3:559-66.

5 Bissell DM. Hepatic fibrosis as wound repair: a progress report. F Gastroenterol 1998;33:295-302

6 Bahr MJ, Vincent KJ, Arthur MJP, et al. Control of the tissue inhibitor of metalloproteinases-1 promoter in culture activated rat hepatic stellate cells: Regulation by activator protein-1 DNA binding proteins. Hepatology 1999;29:83948.

7 Elsharkawy AM, Wright MC, Hay RT, et al. Persistent expression of nuclear factor- $\mathrm{\kappa B}$ in cultured rat hepatic stellate cells involves the induction of potentially novel rel-like factors and prolonged changes in the expression of IKB family proteins. Hepatology 1999;30:761-9.

8 Jan YN, Jan LY. HLH proteins, fly neurogenesis, and vertebrate myogenesis. Cell 1993;75:827-30.

9 Weintraub H. The MyoD family and myogenesis: redunWeintraub H. The MyoD family and myogenesis: red
dancy, networks, and thresholds. Cell 1993;75:1241-4.

10 Olsen EN, Klein WH. bHLH factors in muscle development: dead lines and commitments, what to leave development: dead lines and commitments, wh
in and what to leave out. Genes Dev 1994;8:1-8.

11 Blackwell TK, Weintraub H. Differences and similarities in DNA-binding preferences of MyoD and E2A protein complexes revealed by binding site selection. Science 1990;250 1104-10.
12 Murre C, McCaw PS, Vaessin H, et al. Interactions between heterologous helix-loop-helix proteins generate complexes that bind specifically to a common DNA sequence. Cell 1989;58:537-44

13 Lassar AB, Davis RL, Wright WE, et al. Functional activity of myogenic $\mathrm{HLH}$ proteins requires hetero-oligomerization with E12/E47-like proteins in vivo. Cell 1991;66:305-15.

14 Roberts VJ, Steebergen R, Murre C. Localisation of E2A mRNA expression in developing and adult rat tissues. Proc Natl Acad Sci USA 1993;90:7583-7.

15 Benezra R, Davis RL, Lockshon D, et al. The protein Id: A negative regulator of helix-loop-helix DNA binding proteins. Cell 1990;61:49-59.

16 Iredale JP, Murphy G, Hembry RM, et al. Human hepatic lipocytes synthesise tissue inhibitor
metalloproteinases-1. $\mathcal{F}$ Clin Invest 1992;90:282-7.

17 Iredale JP, Benyon RC, Pickering J, et al. Mechanisms of spontaneous resolution of rat liver fibrosis - hepatic stellate cell apoptosis and reduced hepatic expression of metalloproteinase inhibitors. F Clin Invest 1998;102:538-49.

18 Halzonetis TD, Kandil AN. Determination of the c-Myc DNA-binding site. Proc Natl Acad Sci USA 1991;88:61626.

19 Rockey DC, Boyles JK, Gabbiani G. Rat hepatic stellate lipocytes express smooth muscle actin upon activation in vivo and in culture. F Submicrosc Cytol Pathol 1992;24:193203.

20 Horwitz M. Hypermethylated myoblasts specifically deficient in MyoD autoactivation as a consequence of instability of MyoD. Exp Cell Res 1996;226:170-82.

21 Biggs TE, Cooke SJ, Barton CH, et al. Induction of activator protein-1 (AP-1) in macrophages by human immunodeficiency virus type-1 Nef is a cell-type-specific response that requires both Hck and MAPK signaling events. $\mathcal{F} \mathrm{Mol}$ Biol 1999;290:21-35.

22 Davis RL, Weintraub H, Lassar AB. Expression of a single transfected cDNA converts fibroblasts into myoblasts. Cell 1987;51:987-1000.

23 Ghislaine Mayer DC, Leinwand LA. Sarcomeric gene expression and contractility in myofibroblasts. 7 Cell Biol 1997;139:1477-84.

24 Yokoi Y, Namihisa T, Kuroda H, et al. Immunocytochemical detection of desmin in fat-storing cells (Ito cells). Hepatology 1984;4:709-14.

25 Bataller R, Nicolas JM, Gines P, et al. Arginine vasopressin induces contraction and stimulates growth of cultured human hepatic stellate cells. Gastroenterology 1997;113: $615-24$

26 Wang NP, Marx J, McNutt MA, et al. Expression of myogenic regulatory proteins (Myogenin and MyoD1) in small blue round cell tumors of childhood. Am f Pathol 1995;147:1799-810.

27 Lee KS, Buck M, Houglum K, et al. Activation of hepatic stellate cells by TGF alpha and collagen type 1 is mediated by oxidative stress through $c-m y b$ expression. $\mathcal{F}$ Clin Invest 1995;96:2461-8.

28 Weiner JA, Chen A, Davis BH. Platelet-derived growth factor is a principle inductive factor modulating mannose 6-phosphate/insulin-like growth factor-II receptor gene expression via a distal E-box in activated hepatic stellate cells. Biochem f 2000;345:225-31.

29 Boyd KE, Wells J, Gutman J, et al. c-Myc target gene specificity is determined by a post-DNA binding mechanism. Proc Natl Acad Sci USA 1998;95:13887-92.

30 Miltenberger RJ, Sukow KA, Farnham PJ. An E-boxmediated increase in cad transcription at G1/S-phase boundary is supposed by inhibitory c-Myc mutants. Mol Cell Biol 15:2527-35.

31 O'Regan S, Traiffort E, Ruat M, et al. An electric lobe suppressor for a yeast choline transport mutation belongs to a new family of transporter-like proteins. Proc Natl Acad Sci USA 2000;97:1835-40.

32 Whiteside ST, Epinat JC, Rice NR, et al. IB-, a novel member of the IB family, controls RelA and cRel NF-B activity. EMBO F 1997; 16:1413-26.

33 Monteil A, Mennessier G, Bourinet E, et al. Molecular and functional properties of the human $\alpha(1 \mathrm{G})$ subunit that forms T-type calcium channels. F Biol Chem 2000;275: 6090-100.

34 Abu Hatoum O, Gross-Mesilaty S, Breitschopf K, et al. Degradation of myogenic transcription factor MyoD by the ubiquitin pathway in vivo and in vitro: regulation by specific DNA binding. Mol Cell Biol 1998;18:5670-7.

35 Bounpheng MA, Dimas J, Dodds SG, et al. Degradation of Id proteins by the ubiquitin-proteasome pathway. FASEB $\mathcal{F}$ 1999;13:2257-64.

36 Langlands $\mathrm{K}$, Yin $\mathrm{X}$, Anand $\mathrm{G}$, et al. Differential interactions of Id proteins with Basic-Helix-Loop-Helix transcription factors. $\mathcal{F}$ Biol Chem 1997;272:19785-93. 